Journal for ImmunoTherapy of Cancer

\section{Therapy of lymphoma by immune checkpoint inhibitors: the role of $T$ cells, NK cells and cytokine-induced tumor senescence}

To cite: Ahmetlic F, Fauser J, Riedel T, et al. Therapy of lymphoma by immune checkpoint inhibitors: the role of T cells, NK cells and cytokineinduced tumor senescence. Journal for ImmunoTherapy of Cancer 2021;9:e001660. doi:10.1136/jitc-2020-001660

MR and RM contributed equally. Accepted 10 December 2020

\section{Check for updates}

(c) Author(s) (or their employer(s)) 2021. Re-use permitted under CC BY-NC. No commercial re-use. See rights and permissions. Published by BMJ.

${ }^{1}$ Helmholtz-Zentrum München, Eigenständige Forschungseinheit Translationale Molekulare Immunologie, München, Germany ${ }^{2}$ Helmholtz-Zentrum München, Institut für Molekulare Immunologie, München, Germany

${ }^{3}$ Klinik und Poliklinik für Strahlentherapie und Radioonkologie, LMU München, München, Germany

${ }^{4}$ Klinik für Dermatologie, Eberhard-Karls-Universitat

Tübingen, Medizinische Fakultät, Tübingen, Germany

\section{Correspondence to} Professor Ralph Mocikat; Mocikat@helmholtz-muenchen. de

\section{ABSTRACT}

Background Although antibodies blocking immune checkpoints have already been approved for clinical cancer treatment, the mechanisms involved are not yet completely elucidated. Here we used a $\lambda$-MYC transgenic model of endogenously growing B-cell lymphoma to analyze the requirements for effective therapy with immune checkpoint inhibitors.

Methods Growth of spontaneous lymphoma was monitored in mice that received antibodies targeting programmed cell death protein 1 and cytotoxic $T$ lymphocyte-associated protein-4, and the role of different immune cell compartments and cytokines was studied by in vivo depletion experiments. Activation of $\mathrm{T}$ and natural killer cells and the induction of tumor senescence were analyzed by flow cytometry.

Results On immune checkpoint blockade, visible lymphomas developed at later time points than in untreated controls, indicating an enhanced tumor control. Importantly, $20 \%$ to $30 \%$ of mice were even long-term protected and did never develop clinical signs of tumor growth. The therapeutic effect was dependent on cytokine-induced senescence in malignant B cells. The proinflammatory cytokines interferon- $\gamma$ (IFN- $\gamma$ ) and tumor necrosis factor (TNF) were necessary for the survival benefit as well as for senescence induction in the $\lambda$-MYC model. Antibody therapy improved T-cell functions such as cytokine production, and long-time survivors were only observed in the presence of T cells. Yet, NK cells also had a pronounced effect on therapy-induced delay of tumor growth. Antibody treatment enhanced numbers, proliferation and IFN- $\gamma$ expression of NK cells in developing tumors. The therapeutic effect was fully abrogated only after depletion of both, T cells and NK cells, or after ablation of either IFN- $\gamma$ or TNF.

Conclusions Tumor cell senescence may explain why patients responding to immune checkpoint blockade frequently show stable growth arrest of tumors rather than complete tumor regression. In the lymphoma model studied, successful therapy required both, tumor-directed T-cell responses and NK cells, which control, at least partly, tumor development through cytokine-induced tumor senescence.

\section{BACKGROUND}

The advent of immune checkpoint blockade (ICB) has revolutionized immunotherapy of various types of malignant disease in the clinics. The underlying rationale is to reinvigorate tumor-reactive $\mathrm{T}$ cells, which have been silenced or exhausted in the tumor microenvironment. ${ }^{1}$

It is well established that T-cell inhibitory molecules such as programmed death-1 (PD-1) or cytotoxic T lymphocyte-associated protein-4 (CTLA-4) are frequently upregulated on tumor-infiltrating lymphocytes on chronic stimulation, thereby suppressing stimulatory signals ignited by T-cell receptor or CD28 engagement. ${ }^{2-6}$ Blocking these immune checkpoints by using monoclonal antibodies (mAbs) harnesses T cells to attack cancer by reactivating functions like $\mathrm{T}$ celldependent cytotoxicity.

Ipilimumab, nivolumab and pembrolizumab, which are directed against CTLA-4 and PD-1, respectively, are used for clinical therapy of several types of cancer and have been proven to be highly effective in terms of prolonging patient survival, particularly when administered as a combination regimen. ${ }^{7-9}$ Responding patients, however, rarely show complete tumor eradication, but rather retain residual malignant cells, whose growth seems to be stably arrested for years. ${ }^{9-12}$ This suggested that, beyond T cell-mediated cytolysis, other mechanisms are also involved in conveying the therapeutic effect of ICB therapy.

Previous data indicated that tumor-specific $\mathrm{CD}^{+}$(Th1) cells producing interferon- $\gamma$ (IFN- $\gamma$ ) and tumor necrosis factor (TNF) induce growth arrest of malignant cells. ${ }^{13} 14$ It is further known that IFN- $\gamma$ and TNF can activate the $\mathrm{p} 16^{\mathrm{Ink} 4 \mathrm{a}}-\mathrm{Rb}$ and the $\mathrm{p} 53-\mathrm{p} 21$ 
signaling pathways, ${ }^{12}{ }^{14}$ which induce cellular senescence, that is enduring cell cycle arrest, and that treatment with checkpoint-targeting mAbs promotes IFN- $\gamma$ responses. ${ }^{12}$ In line with these findings, the therapeutic potential of ICB required IFN- $\gamma$-mediated senescence induction in malignant cells that escaped from cytotoxicity, as revealed in two independent mouse models of endogenously arising cancer. ${ }^{12}$

Having shown that combined anti-PD-1 and antiCTLA-4 therapy but not treatment with single mAbs is highly effective in suppressing tumor growth in an endogenous B-cell lymphoma model, ${ }^{1215}$ we set out to investigate which immune cell subpopulations are involved in mediating the therapeutic success and the cytokine-dependent senescence induction in this model. We demonstrate that not only $\mathrm{T}$ cells, but also natural killer (NK) cells play a crucial role for therapy of lymphoma with anti-PD-1 and anti-CTLA-4 mAbs.

\section{METHODS}

\section{Animal experiments}

All mice used were housed under specific pathogenfree conditions in our animal facility. Breeding of mice and all animal experiments were performed in accordance with animal welfare regulations and approved by the responsible authority. Wild-type (wt) C57BL/6 mice were purchased from Taconic (Ry, Denmark), $\lambda$-MYC C57BL/6 mice were bred in our animal facility. ${ }^{15}$ Mice were sacrificed when they had visible tumors. All results were compared with untreated control mice.

For therapy, clinically unapparent $\lambda$-MYC mice received four intraperitoneal injections of a combination of $100 \mu \mathrm{g}$ anti-PD-1 mAb (J43, Bio X Cell, West Lebanon, USA) and $100 \mu \mathrm{g}$ anti-CTLA-4 mAb (UC10-4B9, BioLegend, San Diego, USA) every 10 days starting at day 55 after birth.

Cytokine neutralization in vivo was performed with anti-IFN- $\gamma$ mAb (XMG-1.2, Core Facility mAb, HelmholtzZentrum München) or anti-TNF mAb (XT3.11, Bio X Cell). Intraperitoneal administration of these mAbs started on day 54 with an initial dose of $500 \mu \mathrm{g}$ and was continued in 10-day intervals with $300 \mu \mathrm{g}, 5-6$ hour prior to the anti-PD-1/CTLA-4 mAbs injection. After day 85, mice received $150 \mu \mathrm{g}$ anti-IFN- $\gamma \mathrm{mAb}$ or anti-TNF mAb.

Depletion of $\mathrm{T}$ cells in vivo was done by intraperitoneally injecting anti-MmTC mAb (2G5; Core Facility mAb, Helmholtz-Zentrum München) ${ }^{1617}$ starting at day 54 with an initial dose of $1 \mathrm{mg}$. Subsequently, $400 \mu \mathrm{g}$ anti-MmTC $\mathrm{Ab}$ were applied twice a week. NK cells were depleted by delivering $50 \mu \mathrm{g}$ polyclonal anti-Asialo-GM1 Ab (eBioscience, San Diego, USA) or $100 \mu \mathrm{g}$ TM- $\beta 1$ (Core Facility mAb, Helmholtz-Zentrum München) intraperitoneally in weekly to biweekly intervals starting at day 54 . Successful T-cell or NK-cell depletion was verified by staining splenocytes with fluorochrome-labeled mAbs against CD4 and CD8 for T cells or NK1.1 for NK cells. The efficiency of depletion was $>99 \%$.

\section{Fluorescence-activated cell sorting (FACS)}

Spleens were dissected and homogenized via a $40 \mu \mathrm{m}$ cell strainer in MEM (minimal essential medium). After rinsing with erythrocyte lysis buffer, meshing through a $35 \mu \mathrm{m}$ cell strainer and washing with PBS (phosphatebuffered saline), splenocytes were analyzed by FACS.

$\mathrm{T}$ cells were stained with fluorochrome-labeled $\mathrm{mAb}$ against CD3 (17A2, BD, Heidelberg, Germany), CD4 (RM4-5, eBioscience) and CD8 (53-6.7 and eBioH35-17.2, both eBioscience) for $30 \mathrm{~min}$ at $4^{\circ} \mathrm{C}$. NK cells were defined as NK1.1 $1^{+} \mathrm{CD}^{-} \mathrm{CD} 19^{-}$by staining for NK1.1 (PK136, BD), CD3 and CD19 (1D3, BD). NK cells were further characterized with fluorochrome-conjugated mAbs against PD-1 (J43, eBioscience) and CTLA-4 (UC10-4B9, BioLegend). Viable cells were identified by using the LIVE/DEAD Fixable Blue Dead Cell Stain Kit (ThermoFisher Scientific, Waltham, USA). Intracellular detection of Ki-67 was done using anti-Ki-67 (SolA15, eBioscience), according to the manufacturer's recommendations.

For analysis of intracellular IFN- $\gamma$ and TNF, cells were stimulated for 4 hours with $1 \mu \mathrm{g} / \mathrm{mL}$ PMA (phorbol myristate acetate) and $1 \mu \mathrm{g} / \mathrm{mL}$ ionomycin (both SigmaAldrich, Taufkirchen, Germany) in the presence of $3 \mu \mathrm{g} /$ $\mathrm{mL}$ Brefeldin A (eBioscience). After labeling of surface molecules (CD4, CD8, NK1.1), cells were fixed with IC Fixation Buffer (eBioscience), permeabilized with Permeabilization Buffer (eBioscience) and stained with antiIFN- $\gamma$ (XMG-1.2, BioLegend) or anti-TNF (MP6-XT22, eBioscience) $\mathrm{mAb}$, respectively. Cells were subsequently measured by flow cytometry on an LSR II instrument using DIVA software (both BD) and the results were analyzed with FlowJo software.

\section{Analysis of senescence}

FACS-based measurement of cellular senescence was done as described elsewhere. ${ }^{18}{ }^{19}$ In brief, $10^{6}$ splenocytes were incubated with $100 \mathrm{nM}$ bafilomycin A1 (Tocris Bioscience, Bristol, UK) in serum-free medium for 1 hour at $37^{\circ} \mathrm{C}$ to increase the lysosomal $\mathrm{pH}$ value. Then, 5-do decanoylaminofluorescein-di- $\beta$-galactopyranoside $\quad\left(\mathrm{C}_{12}{ }^{-}\right.$ FDG, Life Technologies, Frankfurt, Germany), which is a chromogenic substrate of senescence-associated $\beta$-galactosidase (SA- $\beta$-gal), was added at a final concentration of $50 \mu \mathrm{M}$, and cells were left for 1 hour at $37^{\circ} \mathrm{C}$ for converting the substrate. Finally, cells were washed with PBS twice, stained for CD19 expression and viability and analyzed by FACS. Senescence was defined on the basis of high $\mathrm{C}_{12} \mathrm{FDG}$ and SSC (side scatter) signals. The gates were defined by using wt splenocytes. Senescence indices were calculated as the x-fold amount of SSC-A $\mathrm{A}^{+} \mathrm{C}_{12} \mathrm{FDG}^{+}$ $\mathrm{B}$ cells related to those from untreated $\lambda$-MYC animals.

To analyze senescence induction in vitro, $0.2 \times 10^{6}$ lymphoma cells were seeded into wells of 96-well U-bottom plates and treated with control medium $(5 \%$ FCS) or a cytokine cocktail containing various concentrations of recombinant mouse IFN- $\gamma$ and TNF (both PeproTech, Hamburg, Germany) for 24 hours at $37^{\circ} \mathrm{C}$. Cellular senescence was detected as described above. 


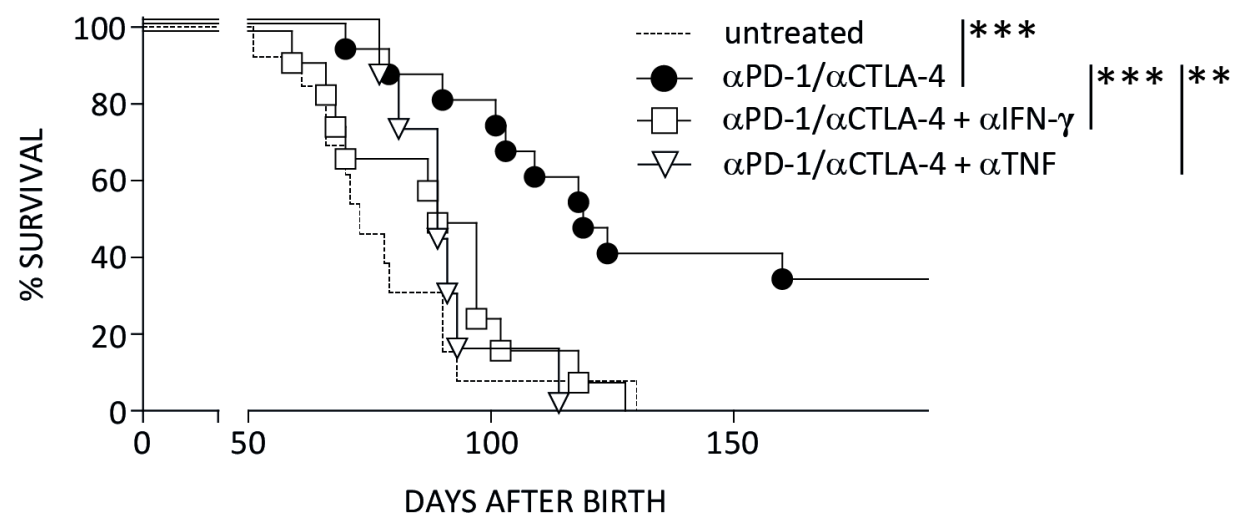

Figure 1 Effect of ICB therapy on tumor growth in $\lambda$-MYC mice. Animals received anti-PD-1 and anti-CTLA-4 mAbs as described in the methods section or were left untreated. Other groups were additionally injected with IFN- $\gamma$-neutralizing and TNF-neutralizing mAbs during therapy. Up to 15 mice were included in each group. For $P$ values see Materials and methods section. CTLA-4, cytotoxic T lymphocyte-associated protein-4; ICB, immune checkpoint blockade; IFN- $\gamma$, interferon- $\gamma$; PD-1, programmed cell death protein 1 .

\section{Analysis of T-cell proliferation}

96-well U-bottom plates were coated with $2 \mu \mathrm{g} / \mathrm{mL}$ antiCD3 (145-2 C11) and anti-CD28 mAb (37N; both from E Kremmer) and incubated overnight at $4^{\circ} \mathrm{C}$. On the next day, plates were washed with PBS and RPMI (Roswell Park Memorial Institute) 1640 medium (10\% FCS), and $0.5 \times 10^{6}$ splenocytes-previously labeled with $10 \mu \mathrm{M}$ Cell Proliferation Dye eFluor 450 (eBioscience)-were seeded into each well. The cells were incubated for 72 hours in RPMI 1640 medium (10\% FCS) in the presence of interleukin-2 (IL-2, $50 \mathrm{U} / \mathrm{mL}$ ). The percentages of proliferating cells were determined by measuring the dilution of eFluor 450 and related to those obtained without CD3/ CD28 stimulation.

\section{NK-cell function in vitro}

NK cells were isolated from spleens of normal mice by immunomagnetic cell separation using the NK Cell Isolation Kit (Miltenyi, Bergisch-Gladbach, Germany). $2 \times 10^{5} \mathrm{NK}$ cells were incubated with YAC-1 cells in a 1:1 ratio in RPMI 1640 medium for 45 hours in the presence of $20 \mathrm{ng} / \mathrm{mL}$ IL-15 (PeproTech). If necessary, antiCTLA-4 and anti-PD-1 mAbs were added to the culture at a concentration of $10 \mu \mathrm{g} / \mathrm{mL}$ each. PD-1 and IFN- $\gamma$ expression of NK cells were then analyzed as described above.

To determine NK-cell degranulation in the presence of NK-sensitive target cells, NK cells were highly enriched from $\lambda$-MYC spleens and again incubated with YAC-1 cells in a 1:1 ratio together with Brefeldin A, Monensin and PE-labeled anti-CD107a mAb (clone 1D4B; BD). After 5 hours, cells were counterstained with anti-NK1.1 and analyzed by FACS.

\section{Statistical analyses}

All results were expressed as means \pm SEM. The unpaired Student's t-test or Mann-Whitney test was used to assess differences between two independent groups. Comparisons of three or more groups were performed with the two-way analysis of variance test followed by Bonferroni multiple comparison test. Survival curves of $\lambda$-MYC mice were compared using the log-rank test. Data analysis was performed with Prism V.5.0 software (GraphPad). The significance levels were depicted as follows: $* \mathrm{p}<0.05$, $* * \mathrm{p}<0.01$ and $* * * \mathrm{p}<0.001$.

\section{RESULTS \\ IFN- $\gamma$-dependent and TNF-dependent senescence induction in B cells is required for lymphoma control by immune checkpoint inhibitors}

For studying therapeutic effects and mechanisms of immune checkpoint inhibition in B-cell lymphoma, we used the $\lambda$-MYC mouse strain, which harbors the $c-M Y C$ oncogene under the control of the B cell-specific immunoglobulin $\lambda$ enhancer. These animals spontaneously develop malignancies, which are located in spleen and lymph nodes and mimic several features of human Burkitt lymphoma. ${ }^{15}$ Clinical symptoms of tumor growth become visible about 60 to 130 days after birth. In earlier disease stages, proliferation of malignant cells remains clinically undetectable. As soon as clinical symptoms appear, tumor masses grow so rapidly that mice have to be euthanized immediately and therapy cannot be started any more. Therefore, $\lambda$-MYC mice received a combined treatment with anti-CTLA-4 and anti-PD-1 mAbs starting before outgrowth of detectable tumor burdens.

Under treatment, (1) tumors developed significantly later and (2) up to $30 \%$ of mice even remained tumorfree for $>200$ days or survived indefinitely (figure 1), as shown recently. ${ }^{12}$ Both effects were completely abrogated when either IFN- $\gamma$-depleting or TNF-depleting mAbs were given during therapy (figure 1). As shown before by using immunohistology, the tumors are infiltrated by $\mathrm{T}$ and B cells and other immune cells. However, the organ architecture is completely destroyed in diseased animals, whereas lymphoid organs from ICB-treated mice that remain healthy show a normal distribution of $\mathrm{T}$ and $\mathrm{B}$ cells and other immune cells. ${ }^{12}$ 
A

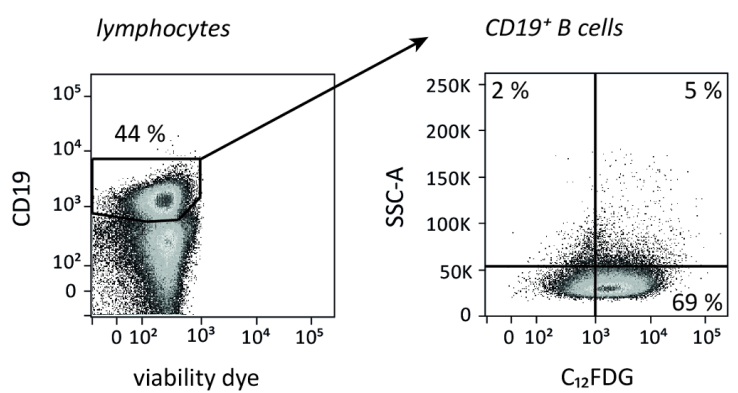

C

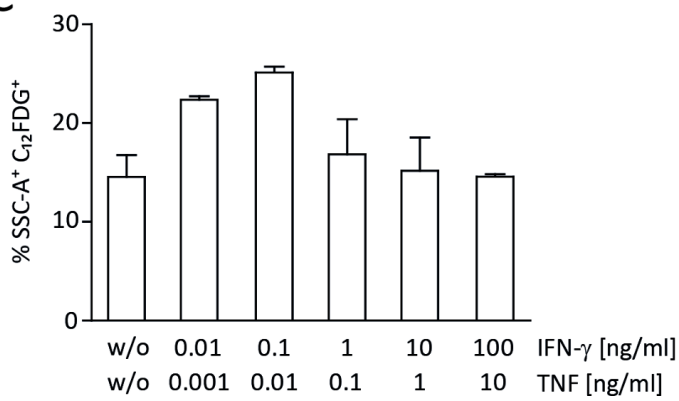

B

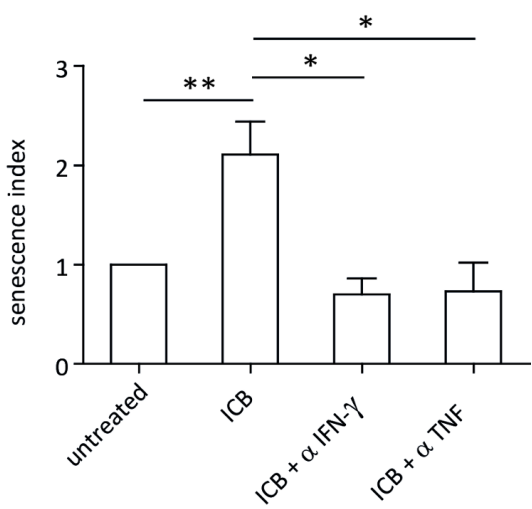

Figure 2 ICB-induced senescence in $\lambda$-MYC tumor cells. (A) Gating strategy for detection of intracellular SA- $\beta$-gal activity in B cells. Analysis of $\mathrm{CD} 19^{+}$cells from a wt mouse is shown as an example. (B) Senescence in $\mathrm{CD} 19^{+}$tumor cells derived from $\lambda$-MYC mice that were or were not treated with ICB mAbs. Part of the treated animals additionally received an IFN- $\gamma$-neutralizing or TNF-neutralizing mAb. The senescence index is defined as the $x$-fold amount of SSC- $A^{+} C_{12} \mathrm{FDG}^{+} \mathrm{B}$ cells related to those from untreated $\lambda$-MYC animals. Four to 12 animals were included in each group. (C) Senescence induction in lymphoma cells from a $\lambda$-MYC mouse in vitro 24 hours after incubation with varying concentrations of IFN- $\gamma$ and TNF. Means and SEM from quadruplicates. ICB, immune checkpoint blockade; IFN- $\gamma$, interferon- $\gamma$; mAbs, monoclonal antibodies; SA- $\beta$-gal, senescenceassociated $\beta$-galactosidase; TNF, tumor necrosis factor.

By immunohistochemical staining of markers like SA- $\beta$-gal and $\mathrm{p} 16^{\text {Ink4a }}$ in combination with Ki67, p21 ${ }^{\text {Cip1 }}$, pHP1 $\gamma$ and H3K9me3 (tri-methylated lysine residue 9 of the histone $\mathrm{H} 3$ protein, which is a senescence marker), we previously showed that IFN- $\gamma$ and TNF can induce senescence, that is, stable growth arrest of malignant cells via the $\mathrm{p} 16^{\text {Ink4a }}$ and p21 pathway. ${ }^{12}$ Accordingly, ICB therapy did not confer a significant survival benefit in p21-deficient MYC mice. ${ }^{12}$ To study the cellular networks involved in ICB-mediated senescence induction in malignant B cells, we now applied a novel approach that relies on flow cytometric detection of intracellular SA- $\beta$-gal activity (figure 2A). ${ }^{18} 19$ During combined anti-CTLA-4/ anti-PD-1 therapy, the frequency of senescent B cells significantly increased in spleens of tumor-developing mice (figure 2B), which confirmed the results obtained by immunohistochemistry. Like the ICB-induced survival prolongation of tumor-developing mice (figure 1), senescence of B cells was also abrogated when either IFN- $\gamma$ or TNF was neutralized in vivo during therapy (figure 2B).

The absence of senescence induction in lymphoma cells by depleting IFN- $\gamma$ or TNF in vivo left the question unanswered whether the combined action of these cytokines is sufficient to induce growth arrest. To address this issue, we performed in vitro experiments using $\lambda$-MYC mouse-derived B cells that were incubated with recombinant IFN- $\gamma$ and TNF. As revealed by SA- $\beta$-galactosidase activity, the presence of the cytokines alone was capable of inducing senescence (figure 2C).

\section{T cells become activated during anti-CTLA-4/anti-PD-1 treatment of B-cell lymphoma}

In the next step, we set out to determine the sources of IFN- $\gamma$ and TNF needed for tumor control and senescence induction in malignant B cells. Since T cells were originally described as the target of ICB, we first studied how the function of this population is affected during treatment of $\lambda$-MYC animals. Even in spleens that were heavily infiltrated with tumor cells despite preceding anti-CTLA-4 and anti-PD-1 therapy, the number as well as the proliferative capacity of both $\mathrm{CD} 4^{+}$and $\mathrm{CD} 8^{+}$cells were significantly increased in comparison to diseased, untreated $\lambda$-MYC animals (figure 3A,B). Importantly, cytokine expression, which was suppressed at least in spleen-derived $\mathrm{CD} 8^{+}$cells from $\lambda$-MYC mice, was enhanced by mAb treatment. The frequency of cells co-expressing IFN- $\gamma$ and TNF significantly increased in the $\mathrm{CD} 4^{+}$and to a lesser degree in the $\mathrm{CD} 8^{+}$compartment (figure 3C).

The data indicate that $\mathrm{T}$ cells were indeed stimulated by the immune checkpoint inhibitors and may be a source of those cytokines that were shown to induce senescence and prolonged survival. ${ }^{14}$ This T-cell activation seems to 
A

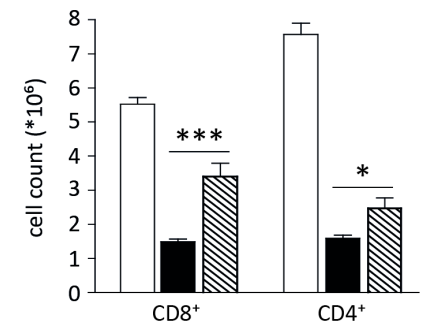

C

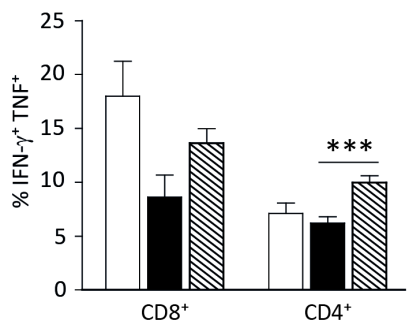

B

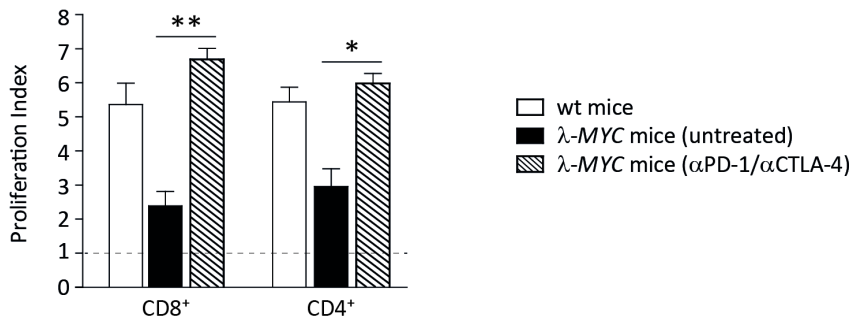

$\mathrm{D}$

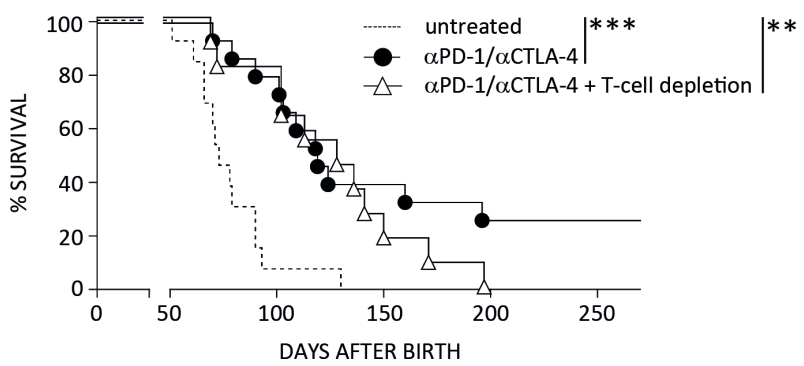

Figure 3 Impact of ICB therapy on T-cell functions. (A) Numbers of CD4 $4^{+}$and $C D 8^{+} \mathrm{T}$ cells, which are reduced in spleens of diseased $\lambda$-MYC mice, recover by ICB therapy. (B) The proliferative capacity of CD4 ${ }^{+}$and $C D 8^{+} \mathrm{T}$ cells in spleens of tumor mice is restored after mAb treatment. Percentages of proliferating cells were measured after CD3/CD28 stimulation in vitro and related to those obtained without stimulation. (C) ICB therapy increases the expression of IFN- $\gamma$ and TNF by CD4 ${ }^{+}$T cells. (D) In the absence of T cells, ICB therapy in $\lambda$-MYC mice fails to produce long-time survivors, but is still effective in delaying tumor development. Eleven to 15 mice were used in each group. CTLA-4, cytotoxic T lymphocyte-associated protein-4; ICB, immune checkpoint blockade; IFN- $\gamma$, interferon- $\gamma$; TNF, tumor necrosis factor.

be functionally relevant because ICB-induced long-term protection beyond day 200 was completely abrogated when mice were depleted of $\mathrm{T}$ cells during therapy. ${ }^{12}$ When monitoring the kinetics of tumor development under ICB therapy and T-cell depletion, however, we noted that the disease-free intervals of $\mathrm{T}$ cell-depleted animals did not differ from those ICB-treated mice that developed tumors in the presence of $\mathrm{T}$ cells (figure 3D). This suggests that, in addition to $\mathrm{T}$ cells, other cell types also contribute to ICB-induced tumor control.

\section{NK cells play a pivotal role for tumor suppression by anti- CTLA-4 and anti-PD-1 mAbs}

We therefore analyzed the role of NK cells during therapy. It is well documented that NK cells of $\lambda$-MYC mice progressively lose effector functions like cytotoxicity and IFN- $\gamma$ expression during the course of disease development. ${ }^{20-23}$ Examining CTLA-4 and PD-1 expression on intratumoral NK cells, we found an upregulation of both molecules (figure 4A), hence an association with the before-mentioned compromised effector functions. Although the upregulation of CTLA-4 was not statistically significant, the data suggest that mAbs used for ICB therapy can directly bind to NK cells with impaired IFN- $\gamma$ expression.

Since NK-cell effector functions in tumor-developing $\lambda$-MYC mice can be partly restored by several means, ${ }^{20}$ we asked whether ICB therapy also reinvigorates NK cells. Compared with untreated control animals of the same age, spleen NK cells from mice that had received ICB therapy showed increased frequencies and enhanced proliferation, although the increment of the latter parameter was not statistically significant (figure 4B,C). Likewise, their cytolytic capacity as measured on the basis of CD107a expression in the presence of an NK sensitive cell line in vitro was slightly increased (figure 4D). It is known, however, that control of $\lambda$-MYC tumors does not necessarily correlate with NK-cell cytotoxicity but rather with IFN- $\gamma$ production by NK cells. ${ }^{23}$ Indeed, the expression of IFN- $\gamma$, which was strictly required for tumor inhibition (figure 1), was elevated in NK cells after ICB therapy. In contrast, TNF production by NK cells remained unaltered (figure $4 \mathrm{E}$ ).

To determine whether the upregulation of IFN- $\gamma$ was a direct effect of blocking CTLA-4 and PD-1 on NK cells, purified NK cells were incubated with the programmed death-ligand 1 (PD-L1)-expressing cell line YAC-1 in vitro in the presence of anti-CTLA-4 and anti-PD-1 mAbs. In this setting, IFN- $\gamma$ expression was significantly enhanced, indicating that other cell populations are not required for ICB-mediated IFN- $\gamma$ induction in NK cells (figure $4 \mathrm{~F}$ ).

\section{Only combined depletion of NK and T cells fully abrogates the therapeutic effect of ICB}

As the ICB-induced prolongation of survival was reversed by IFN- $\gamma$ ablation, we speculated that NK cells, whose IFN- $\gamma$ expression was increased by anti-CTLA-4 and anti-PD-1 mAbs, contribute to the therapeutic effect. Therefore, we 
A

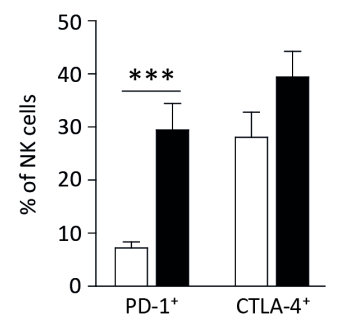

B

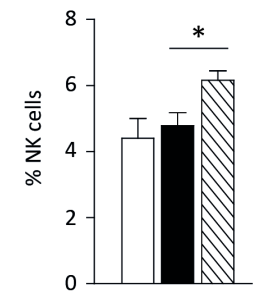

C

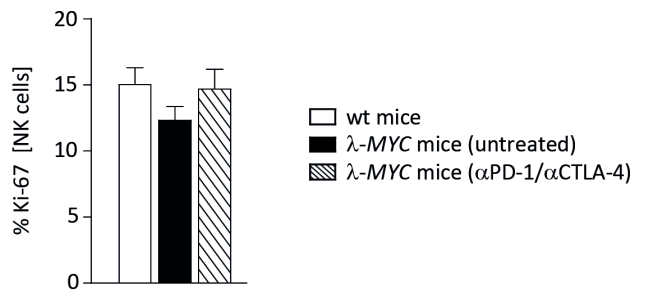

D

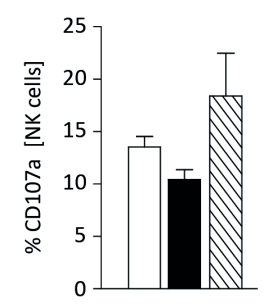

$\mathrm{E}$

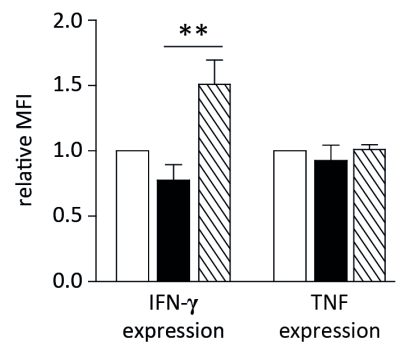

$\mathrm{F}$

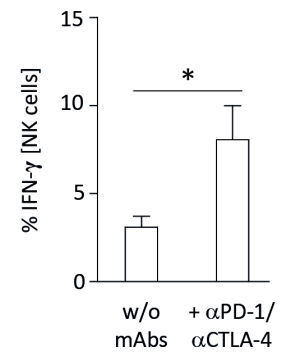

Figure 4 Characterization of spleen NK cells from ICB-treated or untreated $\lambda$-MYC animals. (A) PD-1 and CTLA-4 expression on NK cells is increased in tumor-bearing animals as compared to wt mice. (B) The fraction of NK cells is augmented in spleens from $\lambda$-MYC mice that underwent ICB therapy. (C) NK cells from treated animals exhibit somewhat higher Ki-67 expression. (D) NK cells from treated animals show enhanced degranulation as indicated by increased CD107a surface levels after incubation with YAC-1 cells. (E) IFN- $\gamma$ expression by NK cells, which becomes compromised during the course of tumor development, can partly be restored by ICB therapy. (F) IFN- $\gamma$ expression by NK cells in the presence of YAC-1 cells in vitro is augmented when immune checkpoint inhibitors are added. CTLA-4, cytotoxic T lymphocyte-associated protein-4; ICB, immune checkpoint blockade; IFN- $\gamma$, interferon- $\gamma$; NK, natural killer; PD-1, programmed cell death protein 1 ; TNF, tumor necrosis factor.

performed therapy experiments under selective depletion of NK cells. In the absence of this cell population, tumor growth suppression was partly but not completely abrogated, thus confirming an important functional role of NK cells during therapy. In sharp contrast, ablation of both, NK cells and T cells fully abolished the ICBmediated protection against $\lambda$-MYC lymphoma (figure 5 ).

\section{DISCUSSION}

We could show that combined anti-PD-1 and anti-CTLA-4 treatment is highly effective in treating B-cell lymphoma and that IFN- $\gamma /$ TNF-mediated senescence induction in the malignant B cells contributes to the therapeutic success. Apart from other cytokine-dependent pathways leading to tumor growth inhibition, ${ }^{24-26}$ cytokineinitiated senescence of cancer cells may explain why ICB in patients often leads to long-lasting growth arrest of metastases rather than to complete tumor eradication. ${ }^{9-14}$ In the murine $\lambda$-MYC lymphoma model, we demonstrate

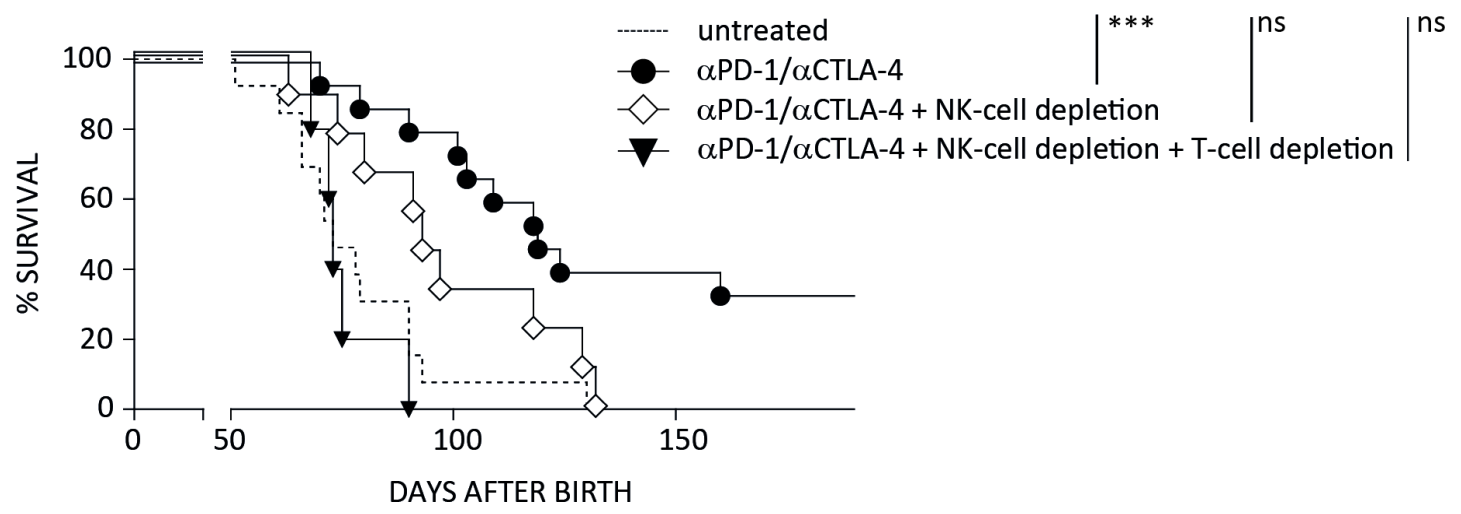

Figure 5 Relevance of NK cells for the success of ICB therapy in $\lambda$-MYC lymphoma. Mice were treated with immune checkpoint inhibitors as described in Materials and methods section. Under simultaneous NK-cell depletion, tumor development is slightly delayed in comparison to the untreated controls, but the difference to this control group is no longer significant. Therapy is completely ineffective only in the absence of NK as well as T cells. Six to 15 mice were included in each group. CTLA-4, cytotoxic T lymphocyte-associated protein-4; NK, natural killer; PD-1, programmed cell death protein 1. 
that not only $\mathrm{T}$ cells are targets of anti-PD-1 and antiCTLA-4 treatment, but that NK cells also play a crucial role for mediating the therapeutic effect.

We earlier reported that the endogenous lymphoma model used here might be more suitable for analyzing tumor-immune interactions than transplantable models. ${ }^{20}{ }^{21}$ The injection of high numbers of lymphoma cells seems to induce NK-cell cytotoxicity and strong IFN- $\gamma$ expression and, eventually, CTL (cytotoxic T lymphocyte) responses. In contrast, an endogenously growing lymphoma progressively suppresses NK-cell and T-cell activity during the course of tumor development. ${ }^{20}{ }^{21}$ Since several pathways of immune escape observed in the $\lambda$-MYC model are therefore not used by transplantable tumors, we suppose that the therapeutic regimen used in our study might also be effective in the latter. Although the beginning of therapy cannot await the development of visible tumor masses (see Results section), the functional data on $\mathrm{T}$ and NK cells reported here might also reflect the situation in clinically apparent tumors because (1) malignant cells were already present when therapy was started and (2) immune cells were also analyzed in those mice that had developed heavy tumor burdens despite preceding therapy.

In tumor-infiltrated spleens of $\lambda$-MYC mice, $T$ cells were identified showing high PD-1 and CTLA-4 levels (T Riedel, F Ahmetlic, unpublished data, 2014) and impaired functional parameters (figure $3 \mathrm{~A}-\mathrm{C}$ ). Interaction with the ligand PD-L1, which is expressed by intratumoral dendritic cells (DCs) and malignant B cells, ${ }^{27} 28$ may therefore drive tumor-reactive $\mathrm{T}$ cells into an inactive state. As predicted by the concept of reactivation of silenced or exhausted tumor-reactive T cells, ${ }^{6}$ treatment with immune checkpoint inhibitors improved T-cell functions like IFN- $\gamma$ and TNF expression and proliferation induced by CD3/CD28 ligation (figure 3B,C). T-cell numbers were also increased (figure $3 \mathrm{~A}$ ), which can be explained by the ameliorated proliferative capacity. Of course, enhanced recruitment of $\mathrm{T}$ cells to the tumor site due to the increment of proinflammatory cytokines cannot be ruled out.

Here we found that this T-cell reactivation was not the only mechanism that was relevant for the in vivo outcome. Though long-time survivors could not be generated by ICB treatment when therapy was done in the absence of $T$ cells, the survival times were still significantly prolonged as compared with untreated mice (figure 3D). After ablation of NK cells, by contrast, the survival benefit in comparison to untreated controls was no longer significant, although not fully abrogated. Therapy was completely ineffective only when ICB was performed under combined ablation of NK and T cells (figure 5).

Generally, activated NK cells are a major source of IFN- $\gamma$, which is necessary to direct T-cell differentiation towards Th1. ${ }^{29}{ }^{30}$ In spleens of $\lambda$-MYC mice, NK cells become activated during tumor development but progressively lose IFN- $\gamma$ expression. ${ }^{20}{ }^{21}$ However, their capability of producing IFN- $\gamma$ can (at least partly) be restored by several stimuli in vivo, for example by Toll-like receptor agonists, ${ }^{20} \mathrm{DCs}^{17}$ or alkalization of the tumor milieu, ${ }^{23}$ strategies that also turned out to prolong survival of $\lambda$-MYC mice. Suppression of $\lambda$-MYC lymphomas always correlated with an increase of IFN- $\gamma$ expression by NK cells, ${ }^{1723}$ but not necessarily with their cytolytic capacity. ${ }^{23}$ Likewise, tumor inhibition by using anti-CTLA- 4 and antiPD-1 mAbs required the proinflammatory cytokines IFN- $\gamma$ and TNF, which were critically needed for delay of tumor growth (figure 1) as well as for senescence induction in $\mathrm{B}$ cells (figure 2B). Direct tumor cell destruction by reactivated NK cells cannot be precluded, because elevated CD107a levels were detected on NK cells derived from mAb-treated mice (figure 4D). An antimetastatic effect of NK cells induced by ICB was also reported in a recent paper, but in contrast to our study, this was only observed in combination with IL-12 treatment. ${ }^{31}$ Improved NK-cell functions were also described as a result of ICB-mediated reinvigoration of $\mathrm{HIV}$-specific $\mathrm{CD} 4^{+} \mathrm{T}$ cells. ${ }^{32}$

As both NK cells and IFN- $\gamma$ were necessary for tumor control and IFN- $\gamma$ expression by NK cells was enhanced by the therapeutic mAbs, we assume that this cell population, besides $\mathrm{T}$ cells, is a crucial source for this cytokine, which subsequently triggers various immunologic and non-immunologic pathways. One of the pathways initiated by NK cell-derived IFN- $\gamma$ eventually gives rise to antitumor T-cell responses. ${ }^{17} 3033$ This may also occur during anti-CTLA-4 and anti-PD-1 therapy apart from direct mAb-induced T-cell activation. Another study indeed suggested that an NK/DC axis has impact on T-cell responses induced by ICB therapy. ${ }^{34}$ However, given the strongly compromised effect of ICB in mice whose senescence pathway was interrupted due to deficient p21 signaling, ${ }^{12}$ cytokine-mediated senescence induction in malignant cells seems to be critically required for the control of $\lambda$-MYC lymphomas. The role of stable growth arrest may have even been underestimated because signaling via $\mathrm{p} 16^{\text {Ink4a }}$, which also mediates senescence, had been unimpaired in the p21-deficient, mAb-treated mice. $^{12}$

In clinical trials, PD-1 blockade has proven useful for therapy of human B-cell malignancies. ${ }^{35}$ However, no benefit was observed by combining PD-1 blockade with mAbs against CTLA- $4 .{ }^{36}$ In the murine $\lambda$-MYC model, by contrast, only the combination but not treatment with single mAbs was effective. ${ }^{12}$ It remains unclear whether this difference may be related to the $\lambda$-MYC tumor entity reflecting human Burkitt lymphoma, which was not included in the clinical study mentioned above.

Taken together, resuscitated $\mathrm{T}$ cells produce elevated amounts of cytokines entailing tumor-suppressive effects like senescence of tumor cells, but they were not exclusively responsible for the prolongation of survival. Thus, it is tempting to speculate that this compartment is more important for completely eradicating tumor cells that may have been driven into growth arrest before, thus providing definitive cure of the disease. Of course, there might exist other cell populations besides $\mathrm{T}$ and NK 
cells that are critical for the therapeutic effect. Thus, we recently showed that DCs are also functionally modulated and thereby have impact on anti-tumor T-cell responses during ICB. ${ }^{37}$ In contrast, macrophages did not show phenotypic or functional changes during ICB treatment in an endogenous tumor model. ${ }^{12}$ Further analyses of the complex alterations of the cellular network during therapy with checkpoint inhibitors are warranted.

Acknowledgements We are grateful to Michael Hagemann and Franziska Liebel for taking care of animal husbandry, Albert Geishauser for genotyping mice and Angela Hoffmann for help in preparing the manuscript. The study includes parts of the doctoral theses of FA, JF, TR and VB at the Ludwig-Maximilians-Universität München.

Contributors FA, JF, TR, VB, CF, NH and EB performed experiments and analyzed data. RH gave advice for measurement of senescence. FA and NH designed the figures. KL supervised experiments. MR and RM conceived and supervised the study and wrote the manuscript.

Funding The work was supported by grants from Deutsche Krebshilfe (70112332, 70112337, 110662 and 110664), Wilhelm-Sander-Stiftung (2012.056.3) and Deutsche Forschungsgemeinschaft (R0 764/15-1 and Cluster of Excellence iFIT, EXC 2180- 390900677).

Competing interests None declared.

Patient consent for publication Not required.

Ethics approval All animal experiments were performed in accordance with German animal welfare legislation.

Provenance and peer review Not commissioned; externally peer reviewed.

Data availability statement All data relevant to the study are included in the article or uploaded as supplementary information. Data are available from the corresponding author.

Open access This is an open access article distributed in accordance with the Creative Commons Attribution Non Commercial (CC BY-NC 4.0) license, which permits others to distribute, remix, adapt, build upon this work non-commercially, and license their derivative works on different terms, provided the original work is properly cited, appropriate credit is given, any changes made indicated, and the use is non-commercial. See http://creativecommons.org/licenses/by-nc/4.0/.

\section{ORCID iD}

Ralph Mocikat http://orcid.org/0000-0003-0141-4937

\section{REFERENCES}

1 Grosso JF, Jure-Kunkel MN. CTLA-4 blockade in tumor models: an overview of preclinical and translational research. Cancer Immun 2013;13:5-17.

2 Walunas TL, Lenschow DJ, Bakker CY, et al. CTLA-4 can function as a negative regulator of T cell activation. Immunity 1994;1:405-13.

3 Freeman GJ, Long AJ, Iwai Y, et al. Engagement of the PD-1 immunoinhibitory receptor by a novel B7 family member leads to negative regulation of lymphocyte activation. $J$ Exp Med 2000;192:1027-34.

4 Collins AV, Brodie DW, Gilbert RJC, et al. The interaction properties of costimulatory molecules revisited. Immunity 2002;17:201-10.

5 Parry RV, Chemnitz JM, Frauwirth KA, et al. CTLA-4 and PD-1 receptors inhibit T-cell activation by distinct mechanisms. Mol Cell Biol 2005;25:9543-53.

6 Wherry EJ. T cell exhaustion. Nat Immunol 2011;12:492-9.

7 Wolchok JD, Kluger H, Callahan MK, et al. Nivolumab plus ipilimumab in advanced melanoma. N Engl J Med 2013;369:122-33.

8 Postow MA, Chesney J, Pavlick AC, et al. Nivolumab and ipilimumab versus ipilimumab in untreated melanoma. $N$ Engl J Med 2015;372:2006-17.

9 Larkin J, Chiarion-Sileni V, Gonzalez R, et al. Combined nivolumab and ipilimumab or monotherapy in untreated melanoma. $N$ Engl $J$ Med 2015;373:23-34

10 Tumeh PC, Harview CL, Yearley JH, et al. PD-1 blockade induces responses by inhibiting adaptive immune resistance. Nature 2014;515:568-71.
11 Topalian SL, Drake CG, Pardoll DM. Immune checkpoint blockade: a common denominator approach to cancer therapy. Cancer Cell 2015;27:450-61

12 Brenner E, Schörg BF, Ahmetlić F, et al. Cancer immune control needs senescence induction by interferon-dependent cell cycle regulator pathways in tumours. Nat Commun 2020;11:1335.

13 Müller-Hermelink N, Braumüller $\mathrm{H}$, Pichler B, et al. TNFR1 signaling and IFN-gamma signaling determine whether $\mathrm{T}$ cells induce tumor dormancy or promote multistage carcinogenesis. Cancer Cell 2008;13:507-18.

14 Braumüller H, Wieder T, Brenner E, et al. T-helper-1-cell cytokines drive cancer into senescence. Nature 2013;494:361-5.

15 Kovalchuk AL, Qi CF, Torrey TA, et al. Burkitt lymphoma in the mouse. J Exp Med 2000;192:1183-90.

16 Kremmer E, Mysliwietz J, Thierfelder S. Induction and suppression of anti-antibodies to syngeneic T cell-binding antibodies in mice. Clin Exp Immunol 1997;109:180-4.

17 Hömberg N, Adam C, Riedel T, et al. CD40-independent natural killer-cell help promotes dendritic cell vaccine-induced T-cell immunity against endogenous B-cell lymphoma. Int $J$ Cancer 2014;135:2825-33

18 Noppe G, Dekker P, de Koning-Treurniet C, et al. Rapid flow cytometric method for measuring senescence associated beta-galactosidase activity in human fibroblasts. Cytometry $A$ 2009;75:910-6.

19 Ernst A, Anders H, Kapfhammer H, et al. HSP90 inhibition as a means of radiosensitizing resistant, aggressive soft tissue sarcomas. Cancer Lett 2015:365:211-22.

20 Brenner CD, King S, Przewoznik M, et al. Requirements for control of B-cell lymphoma by NK cells. Eur J Immunol 2010;40:494-504.

21 Przewoznik M, Hömberg N, Naujoks M, et al. Recruitment of natural killer cells in advanced stages of endogenously arising B-cell lymphoma: implications for therapeutic cell transfer. J Immunother 2012;35:217-22

22 Belting L, Hömberg N, Przewoznik M, et al. Critical role of the NKG2D receptor for NK cell-mediated control and immune escape of B-cell lymphoma. Eur J Immunol 2015;45:2593-601.

23 Pötzl J, Roser D, Bankel L, et al. Reversal of tumor acidosis by systemic buffering reactivates NK cells to express IFN- $\gamma$ and induces NK cell-dependent lymphoma control without other immunotherapies. Int J Cancer 2017;140:2125-33.

24 Qin Z, Blankenstein T. CD4+ T cell--mediated tumor rejection involves inhibition of angiogenesis that is dependent on IFN gamma receptor expression by nonhematopoietic cells. Immunity 2000;12:677-86

25 Koebel CM, Vermi W, Swann JB, et al. Adaptive immunity maintains occult cancer in an equilibrium state. Nature 2007;450:903-7.

26 Kroemer G, Senovilla L, Galluzzi L, et al. Natural and therapyinduced immunosurveillance in breast cancer. Nat Med 2015;21:1128-38.

27 Naujoks M, Weiß J, Riedel T, et al. Alterations of costimulatory molecules and instructive cytokines expressed by dendritic cells in the microenvironment of an endogenous mouse lymphoma. Cancer Immunol Immunother 2014:63:491-9.

28 Riedel T. Intratumorale T-Zellen in einem Spontanlymphommodell der Maus: Aktivierende und supprimierende Mechanismen. Dissertation, Ludwig-Maximilians-Universität München, 2013.

29 Martín-Fontecha A, Thomsen LL, Brett S, et al. Induced recruitment of NK cells to lymph nodes provides IFN-gamma for $\mathrm{T}(\mathrm{H}) 1$ priming. Nat Immunol 2004;5:1260-5.

30 Mocikat R, Braumüller H, Gumy A, et al. Natural killer cells activated by $\mathrm{MHC}$ class I(low) targets prime dendritic cells to induce protective CD8 T cell responses. Immunity 2003;19:561-9.

31 Ohs I, Ducimetière L, Marinho J, et al. Restoration of natural killer cell antimetastatic activity by IL12 and checkpoint blockade. Cancer Res 2017;77:7059-71.

32 Porichis F, Hart MG, Massa A, et al. Immune checkpoint blockade restores HIV-specific CD4 T cell help for NK cells. J Immunol 2018;201:971-81.

33 Adam C, King S, Allgeier T, et al. DC-NK cell cross talk as a nove $\mathrm{CD}^{+}$T-cell-independent pathway for antitumor CTL induction. Blood 2005; $106: 338-44$.

34 Barry KC, Hsu J, Broz ML, et al. A natural killer-dendritic cell axis defines checkpoint therapy-responsive tumor microenvironments. Nat Med 2018;24:1178-91.

35 Xu-Monette ZY, Zhou J, Young KH. PD-1 expression and clinical PD-1 blockade in B-cell lymphomas. Blood 2018;131:68-83.

36 Armand P, Lesokhin A, Borrello I, et al. A phase 1B study of dual PD-1 and CTLA-4 or Kir blockade in patients with relapsed/refractory Iymphoid malignancies. Leukemia 2020. doi:10.1038/s41375-0200939-1. [Epub ahead of print: 29 Jun 2020]. 
37 Scheuerpflug A, Ahmetlić F, Bauer V, et al. The role of dendritic cells for therapy of B-cell lymphoma with immune checkpoint inhibitors.
Cancer Immunol Immunother 2020. doi:10.1007/s00262-020-027676. [Epub ahead of print: 03 Nov 2020]. 